\title{
STUDY OF BLURRING AND HYSTERESIS OF PHASE TRANSFORMATIONS OF MILK FAT BY TRANSIT CALORIMETRY METHOD
}

\author{
Volodymyr Fedorov ${ }^{1}$, Oleg Kepko ${ }^{1}$, Valentyna Kepko ${ }^{2}$, Oleksandr Trus ${ }^{1 凶}$, Svitlana Zhurilo ${ }^{1}$ \\ ${ }^{1}$ Uman National University of Horticulture, 1 Instytutska Str., 20300, Uman, Cherkasy region, Ukraine \\ ${ }^{2}$ Bila Tserkva National Agrarian University, 8/1 Soborna Sq., 09117, Bila Tserkva, Kyiv region, Ukraine \\ 凶alex_trus@ukr.net \\ https://doi.org/10.34302/crpjfst/2020.12.3.8

Article history: $\quad$ ABSTRACT \\ Received: \\ 5 January 2020 \\ Accepted: \\ The so-called effective heat capacity of dairy products, which may include \\ enthalpy changes due to the heat of phase transformations of individual \\ components of the product is used in the technological calculations; the use \\ of the additive rule in this case can lead to the significant errors. The use of \\ Keywords: \\ Milk fat; \\ Heat capacity; \\ Phase transition; \\ Transit colorimetry; \\ Thermoelectric thermostat. \\ the transit calorimetry method described in this article gives an opportunity \\ to deepen the knowledge on the blurring phase transition in dairy products, \\ to clarify information on technological and thermophysical characteristics \\ of the products, to establish a connection between them, to reduce the \\ expenses of energy and material resources per unit of finished products, to \\ detect counterfeit dairy products. The result of the paper is to determine the \\ probable quantitative characteristics of the heat capacity of milk fat of total \\ phase, and the fraction of solid phase due to the phase transformations, their \\ blurring and hysteresis.
}

\section{Introduction}

Technologies for processing, transporting and storing of dairy products require a large amount of different kinds of energy and material resources. Timely and accurate information on the technological and thermophysical characteristics of raw materials, intermediates and finished products during the recipe, technological and process calculations, as well as directly during production contributes to the reduction of expenses of energy, raw materials and materials per unit of finished products.

Milk fat (MF) is the main nutrient and energy component of all whole dairy products, its content in the product is of the main technological characteristics. The existing standardized method for determining the mass fraction of fat in milk, dairy products and canned foods - Gerber's acid method (GOST 5867-90) - requires a certain qualification of a laboratory assistant (the human factor largely influences the accuracy of determination) and significant expenses of time and resources (Ivanova, 2000). Therefore, search for methods of expressdetermination of the proportion of fat and other components of dairy products, for example, on the basis of establishing connections between technological (fat, moisture, acidity, etc.) and thermophysical (heat capacity, thermal conductivity, temperature conductivity, viscosity, etc.) product characteristics is of immediate interest.

Heat capacity is a fundamental thermophysical characteristic for dairy products; there are practically no isothermal or adiabatic processes in this field, when the concept of heat capacity is meaningless and isobaric processes prevail. The heat capacity of the cream should be taken into account, as its temperature changes even when calculating formally mechanical processes, such as the transportation of cream to butter-making equipment.

Heat is a typically extensive characteristic of a process, its relation to a change in body 
temperature due to the absorption of this heat by it, that is, the heat capacity of the body, can also be considered an extensive characteristic, but no longer of the process, but of this body. An additive heat capacity for a substance that is a mixture of components that do not react with each other in a chemical reaction is used in practical calculations.

The so-called effective heat capacity of dairy products, which may include enthalpy changes due to the heat of phase transformations (PT) of individual components of the product is used in the technological calculations; the use of the additive rule in this case can lead to the significant errors. PT occurs this time in a certain temperature range unlike "pure" substances, in addition differently - the product is heated or cooled. In the second half of the last century, this phenomenon was recorded for various substances, including those that remain solid crystalline during the PT, it (phenomenon) is used to study different characteristics of a substance and their interaction with each other. The theory of blurring phase transformations by B. N. Rolov (Rolov and Yurkevich, 1983) is in the basis of these works.

Blurring phase transition (BPT) is the transformation of one condensed phase into another under the conditions of some interval of the actual parameter (temperature, density, concentration, etc.) by definition. According to B. N. Rolov, each thermodynamic characteristic of a complex system can be represented as the sum of the normal part caused by the microstructure of the system and the abnormal part due to the phase transformation. It is a kind of thermodynamic formalism which main task is to give general regularities of change of the abnormal parts irrespective of their specific mechanism.

Phase transformations of a complex nature occur in dairy products during the technological processing, mainly due to the main components of milk fat - triglycerides (modern name triacylglycerides), the difference in melting point and solidification of which can reach 15$20 \mathrm{~K}$ (Belousov, 1984) under the same rate of change of product temperature. Thus, these PT are typical blurring transformations. The use of the transit calorimetry method described in this article gives an opportunity to deepen the knowledge on BPT in dairy products, to clarify information on technological and thermophysical characteristics of the products, to establish a connection between them, to reduce the expenses of energy and material resources per unit of finished products, to detect counterfeit dairy products.

\section{Materials and methods}

The theory of phase blurring transformations (Rolov and Yurkevich, 1983) is widely used in the study of solid-state crystals, solid solutions, etc., that is, when changes in temperature lead mainly to orientational and structural changes in the studied samples, so it is common to call these changes as phase transitions rather than transformations (Aliev, 2007; Yegorov et al., 2008; Yegorov et al., 2009; Yegorov et al., 2013). Such PT are called second-order transitions (PT-2). With regard to dairy products, phase transformations in milk fat occur mainly with a change in aggregate state melting or crystallization (PT-1), although PT-2 also takes place.

In general, PT of seven genera are considered in the solid state physics (Aliev, 2007). PT-3 takes place in ferroelectrics, where temperature rise causes disturbance of the ordered arrangement of dipole moments. PT-4 is a spontaneous magnetization of ferromagnets. If PT-3 and PT-4 occur simultaneously, it is PT-5. PT-6 accompanies superconductivity and superfluidity under extremely low temperatures. PT-7 only takes place in some semiconductors. Phase transitions from PT-3 to PT-7 are considered only if electric and magnetic fields are applied to the appropriate materials.

From a thermodynamic point of view, many physical characteristics of a substance, including heat capacity at the moment of its PT should go to infinity. In real technological processes, the heat capacity $c, \mathrm{~J} /(\mathrm{kg} \cdot \mathrm{K})$ reaches some peak at the moment of PT and then decreases to a new 
value - this is the so-called lambdatransformation. The peak is smoothed for BPT, the point value $T_{o}$ of the PT temperature expands to a certain interval. The dependence of $c(T)$ before and after PT may be different: zero, positive or negative. There is no jump $c(T)$ at $T_{o}$ point, but there is a jump up or down. This dependence is depicted in Figure 1 as positive before and after $T_{o}$ for the perfect jump, real PT1 or PT-2, as well as with the upward jump for BPT.

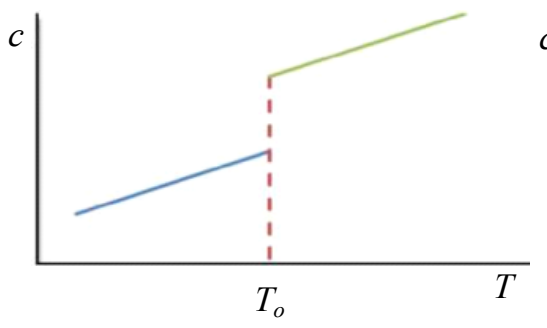

a)

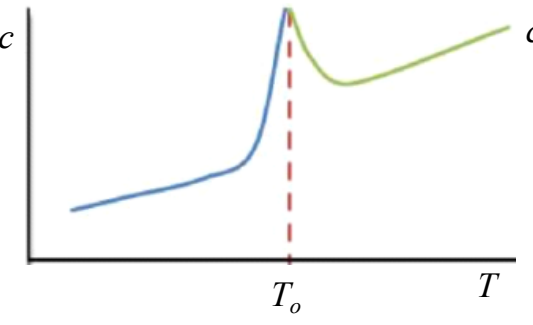

b)

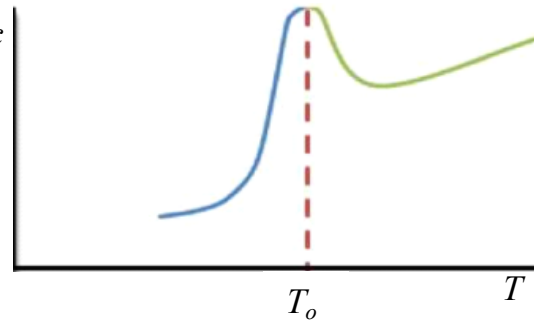

c)

Figure 1. Dependence of heat capacity on temperature under the condition of PT: a) perfect transformation; b) lambda-transformation; c) blurring phase transformation

From a technological point of view, the difference between PT-1 and PT-2 is that the additional energy is released or absorbed during PT-1 and no during PT-2.

Ratio between PT-1 and PT-2 in molecular crystals of various organic compounds was investigated in the works (Yegorov et al., 2008; Yegorov et al., 2009; Yegorov et al., 2013) by one of the methods of differential-and-thermal analysis (DTA) - by differential scanning calorimetry (DSC). We recall that this group of crystals includes crystals of proteins, nucleic acids, binary compounds $\left(\mathrm{CO}_{2}\right.$ and $\left.\mathrm{H}_{2} \mathrm{O}\right)$. Polymorphic transformations also belong to PT of molecular crystals, except typical PT-1 melting, sublimation. All these PTs occur as a rule without breaking the integrity of the molecules. The temperature hysteresis of heat capacity was established for all the test materials, that is, $T_{\text {on }}$ during the samples heating did not coincide with $T_{o o}$ of the samples cooling.

The change of endo- and exopeaks in the work (Yegorov et al., 2008) was used to find out that partial melting, that is PT-1, and polymorphic PT-2 can occur in solid alkanoalcohols, as well as for the quantitative analysis of the temperature dependence of the heat capacity of these compounds on the temperature.
If alcohols with different lengths of finite groups of molecules were the samples in (Yegorov et al., 2008), then diols in (Yegorov et al., 2009), dicarboxylic acids of the same gradation in (Yegorov et al., 2013), conclusions are similar. Information is also given in (Yegorov et al., 2009) on the change of enthalpy and entropy $s$, $\mathrm{J} /(\mathrm{kg} \cdot \mathrm{K})$ of the samples, connection between the value of heat capacity peak $\Delta c$ with heat of PT was established in (Yegorov et al., 2013; Andrianov et al., 1988).

DTA methods are widely used in the determination of $\Delta c, \Delta h, \Delta s$ of loose, viscous and liquid substances. These methods were also used in the study of milk fat, in particular polymorphism phenomena using endopic on thermograms, but the connection between endopic form and "polymorphic characteristics" appeared to be doubtful (Belousov, 1984).

In recent years, DTA methods and other kinds of thermal analysis have been used to evaluate the quality of canned milk (Budanina et al., 2015), to ascertain the connections between the composition of fatty acids of individual MF fractions and their PT temperature (Wang et al., 2017), for the use of MF stearin fraction as a moderator of omega-3 acids oxidation ( $\omega-3)$, which are among the most important nutrients 
(Li et al., 2017), and to establish a connection between heat and PT temperature of individual triacylglycerides (Tolstorebrov et al., 2014).

In general, DTA methods have numerous drawbacks. The keyword in DTA and DSC is "differential", that is all measurements are relative, so their errors are doubled. The thermal resistance of the sample affects not only the overall thermogram level but also the peaks shift, that is the temperature hysteresis of PT (Yegorov et al., 2008). The internal geometry of the sample, especially for the crystalline samples, as well as the external geometry, that is, the volume, surface and the ratio between them influences the reproducibility of the experiments in addition to scanning speed and sample weight.

The study of PT in milk fat with the aim to specify the effects of polymorphic transformations was performed by using DTA, XRSA (X-ray structural analysis), infrared spectral characterization and magnetic resonance spectroscopy, but no single point of view was found on the nature of this effect (Belousov, 1984; Small, 1986).

A significant contribution to the study of the ratio of the solid and liquid phases of fat in dairy products was made by the dilatometric method, since the difference in the specific volumes of these phases is significant. P. Walstra found that the fraction of solid phase $x$ in milk fat (MF) may differ markedly at the same temperature, depending on whether the MF is heated or cooled, that is there is PT hysteresis (Figure 2) (Walstra and van Beresteyn, 1975). Similar hystereses were determined during the cooling and heating of the cream, but the balance "solidliquid MF" required more time (Belousov, 1984).

Works of (Huliaiev-Zaitsev and Tyshchenko, 2003; Tyshchenko, 2002; Upadhyay et al., 2017) were dedicated to detection and prevention of dairy products falsification because of the use of non-dairy fats by the value of Reichert-Messli number and the difference of refractive indexes.

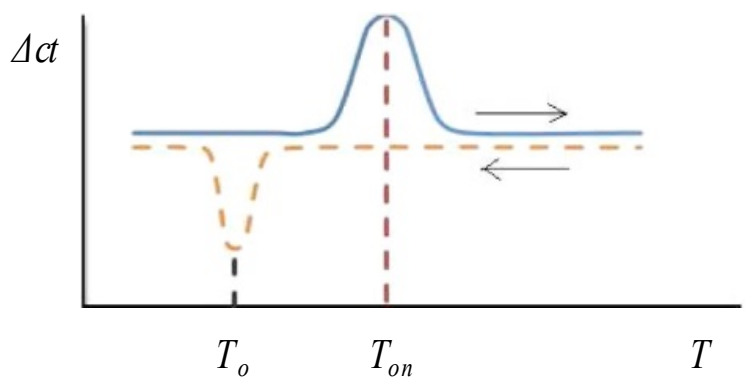

Figure. 2. Temperature hysteresis of the heat capacity of MF

The values of the effective heat capacity of dairy products required for technological calculations were determined by traditional calorimetric methods (direct heating, mixing, etc.) by various researchers, including L. Riegel, K. Becker, V. Leidenfrost, G. Cook, Yu. Olenev, and H. Tverdohleb (cit. (by Fedorov and Pakhomov, 1973) and (Ginzburg et al., 1980)). The differences in heat capacity data increase with the growth of MF in the products, and they can reach one and a half - two times for pure MF. However, information on PT blurring and their hysteresis during heating or cooling is absent everywhere (except for those mentioned above (Small, 1986; Walstra and van Beresteyn, 1975).

These differences can be explained not only by the different composition of dairy products, the content of MF in them in different regions, various seasons, feeding rations and conditions of animals keeping. MF is the most complex of fats of animal and vegetable origin by chemical composition. It is approximately $98 \%$ composed of triacylglycerides, which are composed of more than 140 fatty acids, more than half the weight of MF are saturated acids (from 50.3 to $73.8 \%$ ), the rest are unsaturated, which in turn are divided into mono- and polyunsaturated. The physical properties of triacylglycerides depend not only on the ratio of these acids, but also on how the radicals of the acids are located in the triglyceride. For example, the melting points of triacylglycerides range from $-75^{\circ} \mathrm{C}$ for tributyrin to $+68{ }^{\circ} \mathrm{C}$ for stereodipalmitin. The ability of triacylglycerides to dissolve into each other time in solid state of 
MF and to form mixed crystals during transformation to solid state, their ability to become supercool by $15-20 \mathrm{~K}$ against the crystallization point leads to BPT during the thermal (heating or cooling) processing of dairy products in addition to the aforementioned polymorphism, that is the ability of triacylglycerides of the same composition to form different crystal structures. The mechanical processing of the dairy product and its pre-exposure under certain temperature conditions also promotes the formation of BPT.

A relatively new method of thermal analysis - modulated differential scanning calorimetry (MDSC) is recommended for determining thermal capacity in the paper of (Phinney et al., 2017) dedicated to the possibility of predicting the thermophysical characteristics of new foods depending on the content of major components (fats, hydrocarbons, proteins, fiber, ash and water). At the same time, the sinusoidal heating mode is imposed on the main heating of the sample with linear velocity, which makes it possible to separate the product's own heat capacity from the additional one at the expense of the PT.

The purpose of this work is to develop a method for determining PT and their quantitative characteristics, as well as the heat capacity of MF.

\section{Results and discussions}

3.1. The method of transit calorimetry and its development

This method was developed on the basis of thermometry created in Ukraine and its use in the study of various technological processes (Fedorov, 1974). The heat meter is a plate with a thickness of 1-2 mm and a diameter or side of a square of $8-20 \mathrm{~mm}$, where the junctions of differential thermocouples in the amount of $300-1500$ pieces $/ \mathrm{cm}^{2}$ are led out on the surface. This makes it possible to measure the heat flux density $q, \mathrm{~W} / \mathrm{m}^{2}$ passing through the thermometer up to $3 \%$. The inertia of such heat flow sensors is 5-30 s, which allows recording any changes in heat load on the product sample.

The scheme of transit calorimetry (TCM) (Figure 3) includes heat meters 1 and 2 with thermocouples on the surfaces of sample 3 and sources of supply or removal of heat 4 and 5 .

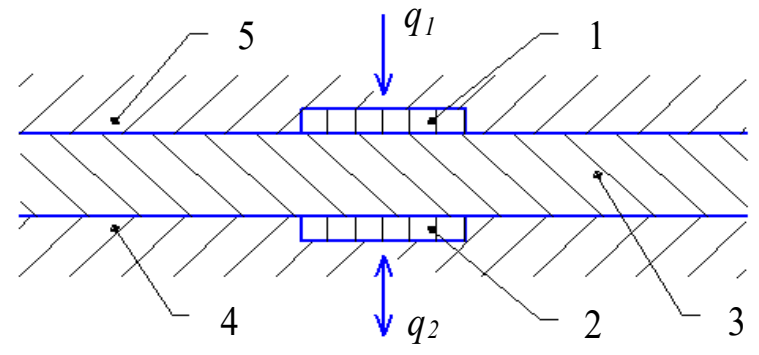

Figure 3. The scheme of transit calorimetry

Thermostatic cameras (TC), or electric heater (E), infrared source (IS) are used as the sources of heat supply, (TC) or thermoelectric (semiconductor) thermostat (TE) for heat removal. Heat supply occurs from above to prevent convection in the liquid sample.

Each experiment begins with a stationary mode which is fixed by the signals of heat meters and thermocouples. The cycle is formed by the perturbation of the mode, $q_{1}$ begins to differ from $q_{2}$, which forms a closed area upon the establishment of a new stationary mode that is proportional to $Q, \mathrm{~J} / \mathrm{m}^{2}$ - the thermal energy accumulated by the sample layer for the transient mode during $\tau$ :

$$
Q=\int_{\tau}\left(q_{1} \pm q_{2}\right) d t
$$

Figure 4 shows the forms of closed surfaces, proportional to $Q$ for the most commonly used combination of TC-TC sources and possible ratios of the initial $q_{n}$ and the final $q_{k}$ of the heat flux density in steady-state modes. The signals $q_{2}$ in Figure 4 are given with the " - " sign for clarity. 


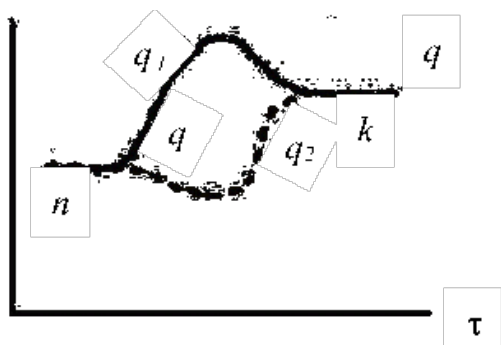

a)

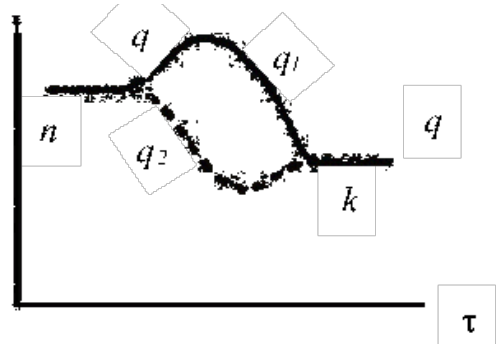

b)

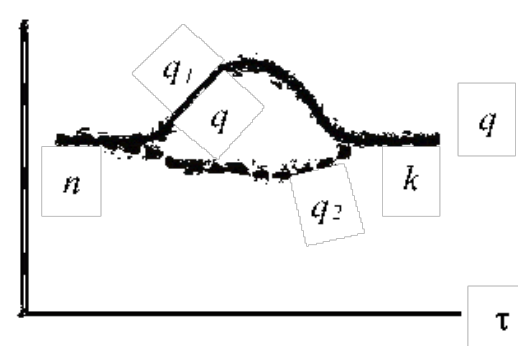

c)

Figure 4. Forms of transient modes (cycles) of TCM

The $Q$ value during one cycle (that is why the method is often called the "cycle method") is proportional to the enthalpy change of the $\Delta h$ sample. The following formula is used to calculate the amount of heat $Q_{o}$ spent for the process conducting if there is no PT in the product during the technological process:

$$
Q_{o}=c m \delta t,
$$

where $\delta t$ is the change of the average temperature of the product per the process, and if PT are significant, then:

$$
Q_{o}=m\left(h_{2}-h_{1}\right),
$$

where $h$ is the enthalpy of the product, its change takes into account both the change of $t$ and the heat of PT. The beginning of the $h$ reference is not fundamental. $h_{0}=0$ at $t=-$ $25^{\circ} \mathrm{C}$ is most often taken for dairy products. The differential equation of thermal conductivity, which is expressed by the enthalpy change is used to derive the calculated formula of the effective heat capacity (Karslow and Eger, 1984):

$$
P \frac{d h}{d \tau}=\frac{\partial}{\partial x}\left(\lambda \frac{d t}{d x}+\lambda \frac{d t}{d y}+\lambda \frac{d t}{d z}\right)+q_{v},
$$

where $q_{v}, \mathrm{~W} / \mathrm{m}^{3}$ is the volume density of the heat flux (due to PT), $\rho, \mathrm{kg} / \mathrm{m}^{3}$ is the product density.

The thermal conductivity $\lambda, \mathrm{W} /(\mathrm{m} \cdot \mathrm{K})$ of dairy and other labile products is highly dependent on the temperature, but (4) can be simplified by using the Kirghoff substitution (Karslow and Eger, 1984):

$$
Q=\frac{1}{\lambda_{\text {mid }}} \int_{t_{1}}^{t_{2}} \lambda d t,
$$

where $t_{1}$ and $t_{2}$ are the initial and final temperatures of the product, $\lambda_{\text {mid }}$ is the average $\lambda$ during the experiment. We get the following by substituting (5) to (4) and simple transformations:

$$
\frac{P}{\lambda_{\text {mid }}} \frac{d h}{d \tau}=\nabla^{2} \theta+\frac{q}{\lambda_{\text {mid }}} .
$$

We obtain the calculated equation for $\Delta h$ by assigning $q$ to the unit of the sample thickness $l$ and taking into account the actual (measured) heat accumulation $q_{1}+q_{2}$ :

$$
\Delta h=\frac{1}{\rho \tau} \int_{\tau}\left(q_{1}+q_{2}\right) d \tau .
$$

It is necessary to know the average temperature increase of the sample over the $\delta t$ cycle to determine $c$ :

$$
c=\frac{\Delta h}{\delta t} .
$$

The found $c$ value is assigned to the average calorimetric temperature of the sample for the $\bar{t}$ cycle: 


$$
\bar{t}=t_{1} \pm \frac{\int_{\tau}\left(t_{2}-t_{1}\right)\left(q_{2}+q_{1}\right) d \tau}{2 \int_{\tau}\left(q_{2}+q_{1}\right) d \tau}
$$

But in most cases there is $\bar{t}=0.5\left(t_{1}+t_{2}\right)$.

The final steady-state mode can be used as the initial one for the next cycle, so the $c(t)$ dependence can be obtained by one experiment over a rather wide temperature range, which is especially important for labile dairy products.

The method of cycles is complex because it is possible to determine the thermal conductivity of the $\lambda$ sample, $\mathrm{W} /(\mathrm{m} \cdot \mathrm{K})$ in each stationary mode:

$$
\lambda=\frac{q l}{\Delta t},
$$

where $q_{1}=q_{2}=q$, and its temperature conductivity $a, \mathrm{~m}^{2} / \mathrm{s}$ :

$$
\alpha=\frac{\lambda}{c \rho} .
$$

This method was used in the study of effective thermal and physical characteristics (TPC) of various foods: beef and pork meat, cattle blood, nonfat greaves, salo (cured pork fat), fruits and vegetables, grain and green malt (Fedorov, 1974; Fedorov, 1987) in addition to dairy products (fat-free milk, dairy cheese, unsweetened condensed milk, cream, etc.).

Work experience with this method allowed minimizing the error in determining the effective heat capacity by optimizing the thickness of $l$ sample (the first independent factor) and the shape of the closed area of the cycle. The measurement accuracy is influenced by this second factor $F$, it was determined by the height of the figure and its length, that is, $\tau_{2}-\tau_{1}=\Delta \tau-$ cycle duration, hence $F=Q / \Delta \tau$. The error itself was taken as the response function, that is, the estimate of the root mean square error of $S$. Molten milk fat in the temperature range of $+10 \ldots+50{ }^{\circ} \mathrm{C}$ was selected as the samples.
The planning and realization of the experiments of the full factorial experiment and then the orthogonal composite plan were carried out; the optimum was determined analytically and graphically, only the optimal values of $F=8.8 \mathrm{~kW} / \mathrm{m}$ and $l=3.75 \mathrm{~mm}$ were obtained.

Microsensors - thermometers with thermocouples with a $5 \mathrm{~mm}$ thickness of dough between them in the middle of a flat pastry piece were placed in the direction of heat flow in the process of baking bread during the study of heatand-mass transfer. The sensors were perforated for free passage of moisture or steam through them.

During the first half of the process, the signals from both thermometers (1 and 2, Figure 5 ) were close to zero, then they formed a closed figure. The signals from the thermometers were then practically the same (curve 3 ). This made it possible to determine the heat capacity of the crumb directly in the baking process; it was $c \rho=0.86 \mathrm{MJ} /\left(\mathrm{m}^{3} \cdot \mathrm{K}\right)$, which is in $\operatorname{good}$ agreement with the reference data (Ginsburg et al., 1980) for the crumb of scone products.

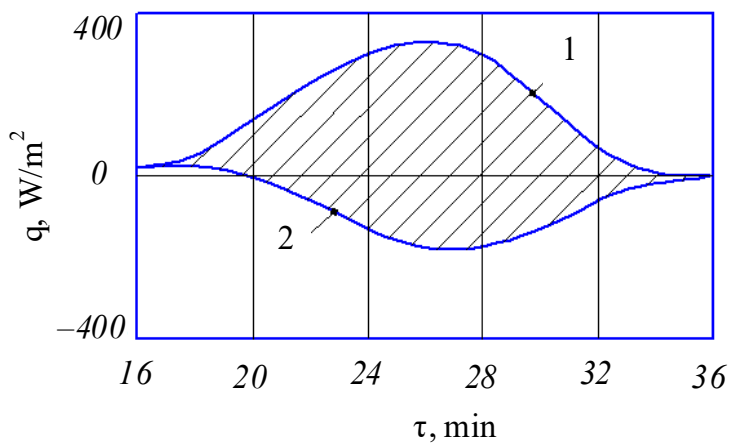

Figure 5. TCM of the fancy pastry in the process of baking

Similar information was obtained during baking of biscuit partly baked product, thermometers with thermocouples were located one (1) on the upper surface of the pastry piece, the second (2) $-5 \mathrm{~mm}$ below it (Figure 6). The signal of the thermometer 1 initially increases sharply, then it drops when the pastry warms, the signal of the thermometer 2 is slow, and then becomes the same. The volumetric heat capacity 
of the pastry at an average temperature of $39^{\circ} \mathrm{C}$ was $c \rho=1.2 \mathrm{MJ} /\left(\mathrm{m}^{3} \cdot \mathrm{K}\right)$, which also corresponds to the reference data (Ginsburg et al., 1980).

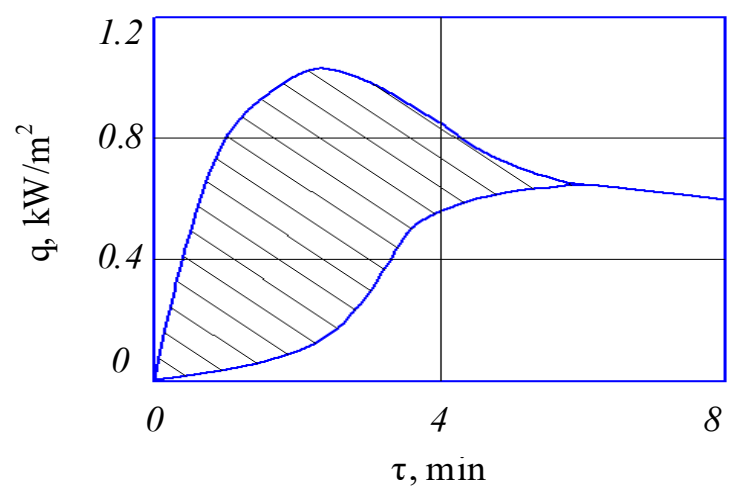

Figure 6. TCM of the biscuit pastry in the process of baking

The TCM method was upgraded by introducing a quasi-stationary or regular mode of the second kind of PP-2 of heating or cooling of the sample in order to continuously obtaining the information needed to determine the heat capacity and other TPC. The heat flux density on both surfaces of the sample was kept constant for this, so that, $q_{1}>q 2$. Then the temperatures of these surfaces will increase in time linearly, with $t_{1}>t_{2}$ (Figure 7), the theory of the method is outlined in (Fedorov, 1987).

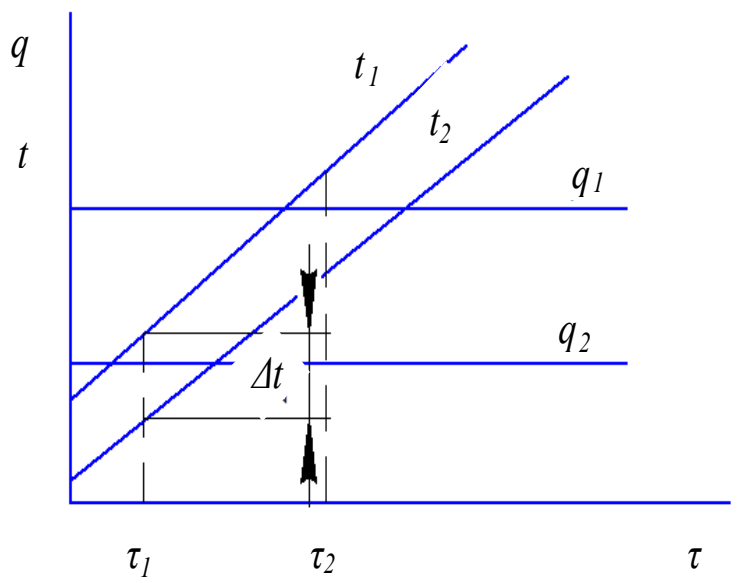

Figure 7. Change in time $q$ and $t$ during PP-2
The calculated formulas for the volumetric heat capacity $c \rho$ and the thermal conductivity $\lambda$ have the form:

$$
\begin{gathered}
c \rho=\frac{q_{1}-q_{2}}{u h} ; \\
\lambda=\frac{q_{1}+q_{2}}{2 \Delta t},
\end{gathered}
$$

where $\Delta t=t_{1}-t_{2} ; u=\Delta t /\left(\tau_{2}-\tau_{1}\right)$.

Methods of cycles and PP-2 are implemented at the same equipment, it is advisable to combine them, if significant PT occur at separate temperature intervals.

The possibility of minimizing the error during PP-2 is presented in (Fedorov, 1987), the results of:

$$
0.17 \leq \frac{q_{1}-q_{2}}{q_{1}+q_{2}} \leq 0.30
$$

\subsection{Results of the study of blurring and hysteresis of the characteristics of milk fat}

According to Rolov's theory, the structure of any whole milk product can be represented as a mixture which ratio between the components depends on the type of the product. It is a mixture of liquid and solid (crystalline) fat for MF (McGee, 1988). This makes it possible to show the effective heat capacity $c$ as the sum of intense (own) $c_{b}$ and additional one by the heat of PT of heat capacity $c_{f}$.

$$
c=c_{b}+c_{f}
$$

$c_{f}$ is meant the amount of heat that emits or absorbs $1 \mathrm{~kg}$ of $\mathrm{MF}$ in a single interval of temperature change. The total heat of $\mathrm{PT}(\mathrm{J} / \mathrm{kg})$ can be defined as the integral of $c_{f}$ in temperature:

$$
L=\int_{t_{n}}^{t} c_{f} d t
$$


Studies have shown that $c_{f}$ may exceed $c_{b}$ for dairy products with a high content of $\mathrm{MF}$; concerning $L$, then this value should not depend on the mode parameters of the technological process and its direction (heating or cooling). The value of $c_{f}$, on the contrary, can significantly depend on these parameters.

TPC and hysteresis of MF characteristics were studied on MF samples with an iodine value of 37.0 and a moisture content of not more than $0.3 \%$. A wide range of temperatures (from minus $60^{\circ} \mathrm{C}$ to plus $80^{\circ} \mathrm{C}$ ) was ensured by the use of different designs of TPC devices; the ETC scheme was used in the range of $60{ }^{\circ} \mathrm{C} . . .+20{ }^{\circ} \mathrm{C}$ supplemented with copper fins which was immersed in the Dewar flask with liquid nitrogen. The intensity of heating load on the sample and its temperature was changed by regulating the proportion of nitrogen boil-off. At positive temperatures, the E-TC scheme was supplemented with the TC-TC scheme with plates with thermometers and thermocouples connected with each other with the gap as the sample thickness. Fat or other pasty or liquid product is placed between the plates (this is a sample) and around them. The use of such joint cassettes allows the sample to be removed from the heat treatment device without disturbing the structure, and to study several samples at a given mode at the same time. Both types of devices were used in the zone of active PT. The error in determining the effective heat capacity was not higher than $\pm 5 \%$.

Figure 8 shows the results of study $c$ in the range of $t=-60 \ldots+80{ }^{\circ} \mathrm{C}$ under the condition of slow cooling of the sample at a rate of $0.003 \mathrm{~K} / \mathrm{s}$, as well as under the condition of heating at the same rate of rapidly pre-cooled sample.

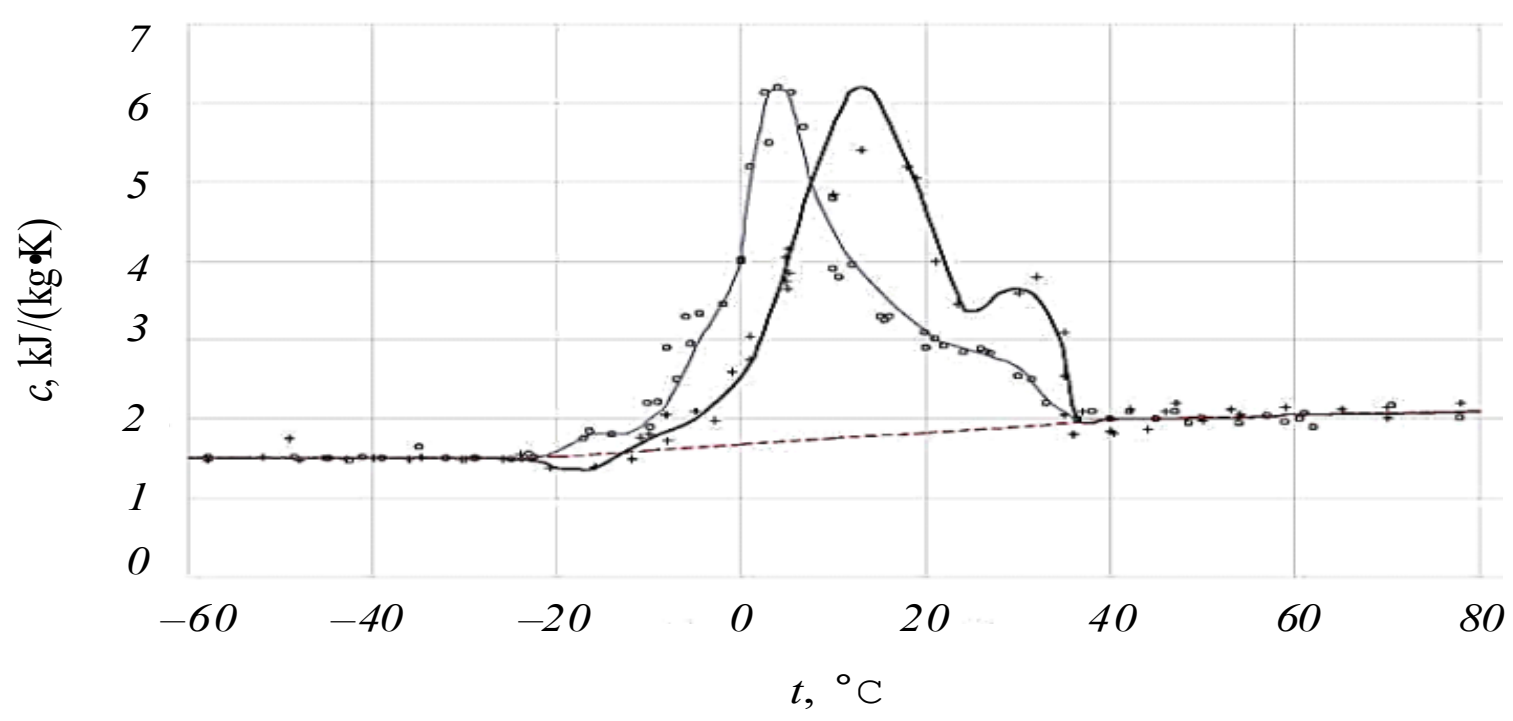

Figure 8. Determination of the effective thermal capacity of MF by the TCM method: "+" - heating, "o" - cooling

The heat capacity of the solid MF is not almost independent of the temperature and it is $c=1.468 \pm 0.009 \mathrm{~kJ} /(\mathrm{kg} \cdot \mathrm{K})$ in the range of $72 \ldots-25^{\circ} \mathrm{C}$. A slight decrease of $c$ compared to the solid state was observed during heating from -20 to $-10^{\circ} \mathrm{C}$, it is probably due to the appearance of PT with an exothermic effect. In the $-25 \ldots+37^{\circ} \mathrm{C}$ range, there are two peaks:
$6.3 \mathrm{~kJ} /(\mathrm{kg} \cdot \mathrm{K})$ at $15^{\circ} \mathrm{C}$ and $3.7 \mathrm{~kJ} /(\mathrm{kg} \cdot \mathrm{K})$ at $30{ }^{\circ} \mathrm{C}$ and one peak during cooling $6.53 \mathrm{~kJ} /(\mathrm{kg} \cdot \mathrm{K})$, almost the same as during heating, but shifted by $10 \mathrm{~K}$ to the left, that is at $+5{ }^{\circ} \mathrm{C}$. This hysteresis occurs throughout the PT range. Additional studies of MF with iodine numbers of 30.9 and 41.9 showed that the magnitude of the peaks shift depends on the 
content of unsaturated acids in the fat. This dependence results in complexes with different energy being formed during melting and curing.

The heat capacity of completely melted fat does not depend on whether it is heated or cooled (Figure 8), it depends on the temperature linearly and in the temperature range of $+37 \ldots+80^{\circ} \mathrm{C}$ :

$$
c=1.7613+0.00586 \pm 0.156 .
$$

A sufficiently large value of error means that BPT have significant values in fully melted fat. The use of TCM for studies of dairy products with different contents of MF has shown that the more water in the product, the less blurring of PT, and they disappear for skim milk.

This method makes it possible to obtain a value for the proper conducting of the technological process, characterization - the content of the solid phase $m_{m}$ in fat:

$$
x=m_{m} / m,
$$

where $m$ is the mixture mass of solid and liquid fat.

Until now, the value of $x$ was determined indirectly by calorimetric or dilatometric methods, since direct phases separation is a long process and is not suitable for production control. Both methods predict extrapolation of dependences $h(t)$ or $v(t)$ of the solid and liquid fat in the PT area. Since both of these dependencies are nonlinear and the extrapolation is linear, the error in determining $x$ by both methods lies in the range of $13-25 \%$.
The TCM method gives the possibility to reduce these errors drastically. If we integrate (14) over a temperature in the range from the lower limit of PT $t_{h}$ to the upper limit of $t_{b}$, we obtain:

$$
\int_{t_{h}}^{t_{b}} c d t=\int_{t_{h}}^{t_{b}} c_{b} d t+\int_{t_{h}}^{t_{b}} c_{f} d t
$$

The left part (19) is the enthalpy difference $h_{b}-h_{h}$ at the range of PT $t_{b}-t_{h}$. Studies have shown that this difference does not depend on the chemical composition of MF and the method of sample preparation - hysteresis is located in the middle of the range of $t_{b}-t_{h}$, the first summand of the right part (19) can be calculated through the heat capacity of the solid $c_{s}$ and liquid $c_{l}$ parts:

$$
c_{f}=x c_{s}+(1-x) c_{l} \text {. }
$$

By integrating (18) over $t$, we obtain that the first summand of the right part is not practically independent on the form of function $x(t)$, hence it is clear that the second summand equal to $L$ must be invariant, which confirms the conclusions of other researchers that $L$ can be considered a constant value. The diagram $c_{f}(t)$ for $M F$ with iodine number of 37 was constructed using (17) and linear interpolation into the BPT area (Figure 9). Determining the area under the lines $c_{f}(t)$ under the conditions of heating and cooling of these samples, as well as fat with other iodine numbers gave a value of $L$ close to $84 \mathrm{~kJ} / \mathrm{kg}$. 


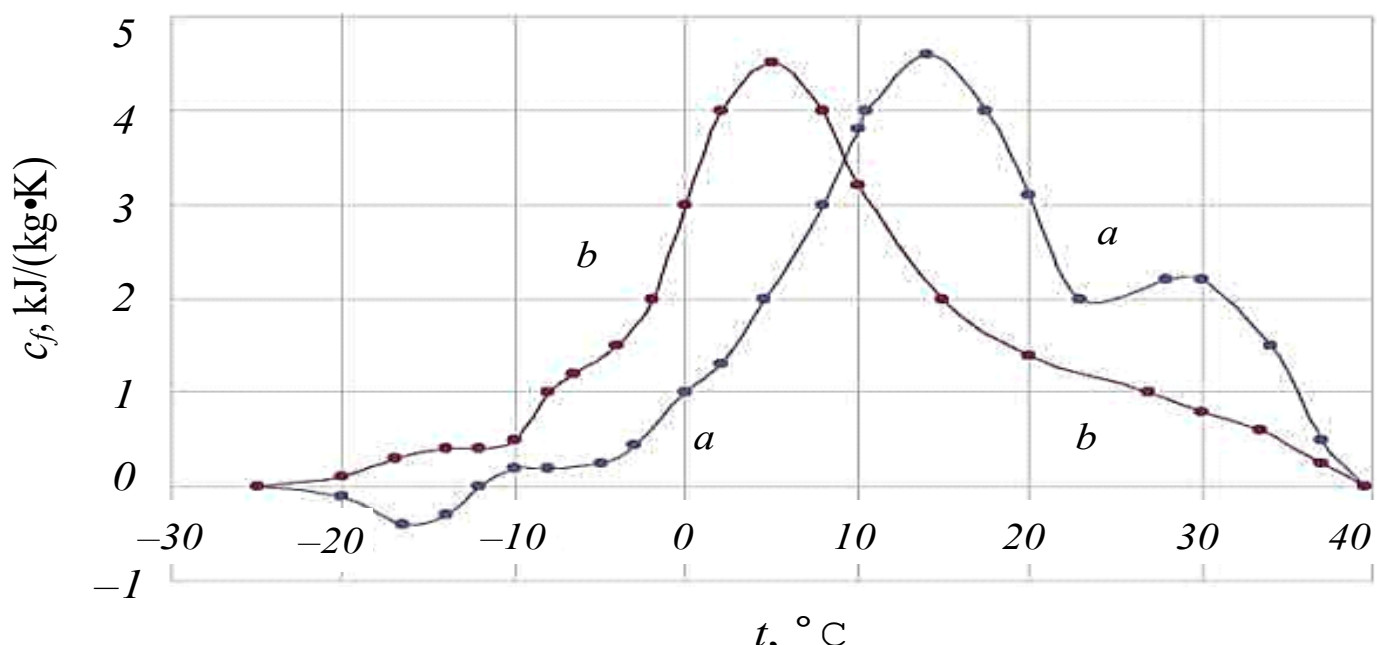

Figure 9. Diagram $c_{f}(t)$ for $\mathrm{MF}$ in $\mathrm{PT}$ area: $\mathrm{a}$ - heating, $\mathrm{b}$ - cooling

The fraction of hardened fat $x$ at any $t$ from $t_{h}$ or $t_{k}$ required for the technological calculations is possible to calculate by using the dependence $c_{f}(t)$ from Figure 9:

$$
x=\int_{t_{\rho}}^{t} \frac{c_{f} d t}{L}=L \int_{t}^{t_{k}} \frac{c_{f} d t}{L} .
$$

You can also use the dependences $c_{b}(t)$ and $h_{k}(t)$ to determine $x(t)$ :

$$
x=\frac{h_{k}-h_{t}-\int_{t}^{t_{k}} c_{b d t}}{h_{k}-t_{\rho}-\int_{t_{\rho}}^{t_{k}} c_{b} d t} .
$$

Figure 10 shows the dependence $x(t)$ for MF with iodine number of 37 , as well as $h(t)$ with $h=0$ at $t=-25^{\circ} \mathrm{C}$ currently accepted in the dairy industry, that is, $h-x-t$ diagram in the field of phase transformations. You can determine the fraction of hardened fat $x$ using this diagram, also the amount of heat that must be removed from $1 \mathrm{~kg}$ of fat to change $x$ for a given value from $x_{1}$ to $x_{2}$, that is $\delta h=h_{x l}-h_{x 2}$.

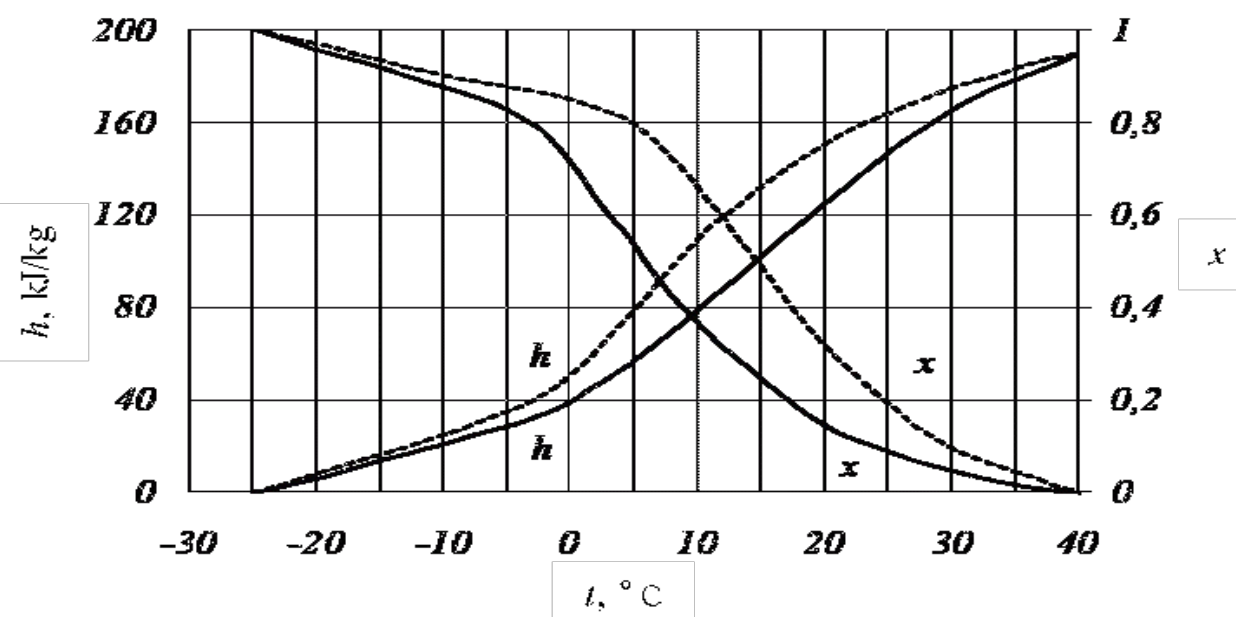

Figure 10. Diagram $h-x-t$ for MF in PT area: "_" - heating, "--“- cooling 
Statistical processing of all the data obtained from the blurring and hysteresis of phase transformations of milk fat using TCM was performed by the methods of mathematical planning of experiments. The main tasks of this processing were to establish a reliable probability and to calculate the magnitude of the PT hysteresis during heating (or mechanic processing) of milk fat, to calculate the PT blurring for these processes as a maximum difference of the heat capacity values within one process, as well as to smoothen experimental data.

Both types of TCM - the method of cycles and the method of quasi-stationary mode, - in accordance with the objectives of their implementation in this work, were carried out as a one-factor experiment, and the aforementioned minimization of the error of TPC measurement of food products by the methods of TCM - was carried out as a multifactorial experiment. This gives the possibility to solve not only interpolation but also optimization problems using TCM methods. The data obtained in this work were used to process the results of the processes study connected with the inversion of heat fluxes during heat treatment of food products (Fedorov et al., 2014).

$\gamma=0.95$ was taken as a reliable probability $\gamma$ of the results of the statistical processing taking into account the magnitude of the main permissible error for the devices implementing
TCM, as well as the ratio of the dynamic properties of the devices with the PT dynamics of milk fat. All the accumulated information from BPT of milk fat obtained by the TCM method was processed in order to solve three main tasks. It was necessary to establish the facts of a statistically significant difference between the sample data of heating and cooling, and vice versa - the belonging of this data of each sample to one group, in spite of BPT. The third task was to smooth out the experimental data, because, as it is clear from the above, there can be no two derivatives at each point of the generalizing curves, but only one, in other words, the lambdaPT for MF is impossible.

The Fisher-Snedecor and Student's distributions, respectively, were used to solve the first two problems, and the method of the moving average (Walkenbach, 2015) was used for the third task. Computer processing was performed using the programs of $\mathrm{F}$ and t-tests, respectively, as well as the Moving in the middle program (Walkenbach, 2015).

Both null hypotheses with a reliable probability of 0.95 were confirmed, the results of processing are shown in Figure 9 and 10, as well as in Table 1 and 2. The curves in Figure 5 and 6 did not require the smoothing of the sensor signals. Tables 1 and 2 are compiled by the program of reducing the number to three significant figures.

Table 1. Characteristics of milk fat

\begin{tabular}{|l|c|c|c|c|c|c|c|c|}
\hline \multicolumn{1}{|c|}{ Temperature } & $\boldsymbol{t},{ }^{\circ} \mathbf{C}$ & $\mathbf{- 6 0}$ & $\mathbf{- 5 0}$ & $\mathbf{- 4 0}$ & $\mathbf{- 3 0}$ & $\mathbf{- 2 0}$ & $\mathbf{- 1 0}$ & $\mathbf{0}$ \\
\hline Heat capacity, heating & $c, \mathrm{~kJ} /(\mathrm{kg} \cdot \mathrm{K})$ & 1.49 & 1.49 & 1.49 & 1.49 & 1.32 & 1.70 & 2.72 \\
\hline Heat capacity, cooling & $c, \mathrm{~kJ} /(\mathrm{kg} \cdot \mathrm{K})$ & 1.49 & 1.49 & 1.49 & 1.49 & 1.68 & 2.14 & 3.96 \\
\hline Blurring, heating & $P, \mathrm{~kJ} /(\mathrm{kg} \cdot \mathrm{K})$ & 0.12 & 0.22 & 0.10 & 0.10 & 0.14 & 0.24 & 0.18 \\
\hline Blurring, cooling & $P, \mathrm{~kJ} /(\mathrm{kg} \cdot \mathrm{K})$ & 0.16 & 0.14 & 0.08 & 0.06 & 0.10 & 0.08 & 0.06 \\
\hline Hysteresis & $H, \mathrm{~K}$ & 0 & 0 & 0 & 0 & 10.0 & 6.0 & 6.2 \\
\hline \hline \multicolumn{1}{|c|}{ Temperature } & $\boldsymbol{t},{ }^{\circ} \mathbf{C}$ & $\mathbf{+ 1 0}$ & $\mathbf{+ 2 0}$ & $\mathbf{+ 3 0}$ & $\mathbf{+ 4 0}$ & $\mathbf{+ 5 0}$ & $+\mathbf{6 0}$ & $+\mathbf{7 0}$ \\
\hline Heat capacity, heating & $c, \mathrm{~kJ} /(\mathrm{kg} \cdot \mathrm{K})$ & 5.74 & 4.72 & 3.82 & 1.98 & 2.02 & 2.1 & 2.24 \\
\hline Heat capacity, cooling & $c, \mathrm{~kJ} /(\mathrm{kg} \cdot \mathrm{K})$ & 4.16 & 3.06 & 2.76 & 1.98 & 2.02 & 2.16 & 2.24 \\
\hline Blurring, heating & $P, \mathrm{~kJ} /(\mathrm{kg} \cdot \mathrm{K})$ & 0.78 & 0.42 & 0.22 & 0.10 & 0.10 & 0.08 & 0.18 \\
\hline Blurring, cooling & $P, \mathrm{~kJ} /(\mathrm{kg} \cdot \mathrm{K})$ & 0.20 & 0.32 & 0.08 & 0.10 & 0.08 & 0.12 & 0.18 \\
\hline Hysteresis & $H, \mathrm{~K}$ & 10.0 & 9.2 & 14,0 & 0 & 0 & 0 & 0 \\
\hline
\end{tabular}


Table 2. Characteristics of milk fat in PT area

\begin{tabular}{|l|c|c|c|c|c|c|c|}
\hline \multicolumn{1}{|c|}{ Temperature } & $\boldsymbol{t},{ }^{\circ} \mathbf{C}$ & $\mathbf{- 2 0}$ & $\mathbf{- 1 0}$ & $\mathbf{0}$ & $\mathbf{+ 1 0}$ & $\mathbf{+ 2 0}$ & $+\mathbf{3 0}$ \\
\hline Heat capacity due to PT, heating & $c, \mathrm{~kJ} /(\mathrm{kg} \cdot \mathrm{K})$ & -0.15 & 0.12 & 0.98 & 3.12 & 2.70 & 1.82 \\
\hline Heat capacity due to PT, cooling & $c, \mathrm{~kJ} /(\mathrm{kg} \cdot \mathrm{K})$ & 0.10 & 0.42 & 2.48 & 2.60 & 1.22 & 0.66 \\
\hline Enthalpy of heating & $h, \mathrm{~kJ} / \mathrm{kg}$ & 6.20 & 20.4 & 40.8 & 80.0 & 128 & 168 \\
\hline Enthalpy of cooling & $h, \mathrm{~kJ} / \mathrm{kg}$ & 8.00 & 20.6 & 51.4 & 109 & 138 & 178 \\
\hline Fraction of solid fat, heating & $X$ & 0.98 & 0.94 & 0.88 & 0.72 & 0.12 & 0.02 \\
\hline Fraction of solid fat, cooling & $X$ & 0.09 & 0.96 & 0.94 & 0.71 & 0.12 & 0.09 \\
\hline
\end{tabular}

\section{Conclusions}

The probable quantitative characteristics of the total heat capacity of milk fat, and due to the phase transformations, the fraction of solid phase, their blurring and hysteresis were determined for the first time. According to the research results, it is desirable to replace and supplement the information on this subject in the reference editions.

\section{References}

Aliev, S. (2007). Blurring of phase transitions in semiconductors and high-temperature superconductors. Institute of Physics of NAS of Azerbaijan. Baku: ELM, 298.

Andrianov, Yu. P., $\quad$ Vyshemirsky, F. A., Kacherauskis, D. V., Klimov, V. P. (1988). Production of dairy butter: Reference book. Ed. by Vyshemirsky, F. A. Moscow: Agropromizdat, 330.

Belousov, A. P. (1984). Physical-and-chemical processes in the production of butter by whipping cream. Moscow: Light and food industry, 264.

Budanina, L. N., Vereshchagin, A. L., Bychin, N. V. (2015). Application of the DSC method for the identification of canned dairy products. Technique and technology of food production, 2(37), 98-104.

Karslow, G., Eger, D. (1984). Thermal conductivity of solids. Moscow: Nauka, 488.

Fedorov, V. H. (1974). Thermometry in food industry. Moscow: Food Industry, 176.

Fedorov, V. H. (1987). Fundamentals of thermal massometry. Kyiv: Vyshcha school, 184.

Fedorov, V., Kepko, O., Skarboviychuk, O. (2014). Returning heat flow during thermal treatment of food. Ukrainian Journal of Food Science, 2(1), 118-123.

Fedorov, V. H., Pakhomov, V. N. (1973). Study of the heat capacity of milk fat. Ann. on scientific-and-research works in the universities of the USSR in the collection of books. Food industry, 10, 57-58.

Ginzburg, A. S., Gromov, M. A., Krasovskaya, G. I. (1980). Thermophysical characteristics of food products. Reference book. Moscow: Food Industry, 288.

Huliaiev-Zaitsev, S., Tyshchenko, L. (2003). Methods for determining of non-milk fats in butter. Standardization, certification, quality, 6, 28.

Ivanova, V. L. (2000). Milk and milk products. Regulatory documents: Refence book. In $3 \mathrm{v}$. Gen. ed. by Ivanova, V.L. Lviv: "Leonorm" Scientific Information Center, 2, 100-111.

Karslow, G., Eger, D. (1984). Thermal conductivity of solids. Moscow: Nauka, 488.

Li, B. Z., Truong, T., Bhandari, B. (2017). Crystallization and melting properties of mixtures of milk fat stearin and omega-3 rich oils. Food Chem, 218, 199-206.

McGee. (1988). On food and cooking. New York: Macmillan Publ. Co., 684.

Phinney, D. M., Frelka, J. C., Heldman, D. R. (2017). Composition-based prediction of temperature-dependent thermophysical food properties: Reevaluating component groups and prediction models. Journal of food science, 82(1), 6-15.

Rolov, B. N., Yurkevich, V. E. (1983). Physics of blurring phase transitions. Rostov: Rostov University Press, 320. 
Small, D. M. (1986). The physical chemistry of lipids. N.Y. London: Plenum Press, 450.

Tolstorebrov, I., Eikevik, T. M., Bantle, M. A. (2014). DSC determination of phase transitions and liquid fraction in fish oils and mixtures of triacylglycerides. Food research international, 58, 132-140.

Tyschenko, L. M. (2002). On the method for identification of milk fat. Bulletin of agrarian science, 3, 67-69.

Upadhyay, N., Goyal, A., Kumar, A., Lal, D. (2017). Detection of adulteration by caprine body fat and mixtures of caprine body fat and groundnut oil in bovine and buffalo ghee using differential scanning calorimetry. International Journal of Dairy Technology, 70(2), 297-303.

Walkenbach, D. (2015). Microsoft Excel 2013. User Bible. M., St. Petersburg, K.: Dialektika, 928.

Walstra, P., van Beresteyn, E. C. H. (1975). Crystallization of milk fat in the emulsified state. Netherlands Milk and Dairy Journal, 29(1), 35-65.

Wang, Y., Li, Y., Han, J., Li, Y., Zhang, L. (2017). Effect of Melting Point on the Physical Properties of Anhydrous Milk Fat. IOP Conference Series: Materials Science and Engineering, 274(1), 012072.

Yegorov, V. M., Marikhin, V.A., Myasnikova, L. P. (2008). Phase transitions in molecular crystals of n-alkano-alcohols. Solid State Physics, 50(1), 123-129.

Yegorov, V. M., Marikhin, V. A., Myasnikova, L. P. (2013). Phase transitions in molecular crystals of dicarboxylic acids. Solid State Physics, 55(5), 975-980.

Yegorov, V. M., Marikhin, V. A., Myasnikova, L.P., Nakamura, N. (2009). Features of phase transitions in molecular crystals of diols. Solid State Physics, 51(10), 2006-2011.

\section{Acknowledgment}

The authors are grateful in the memory of Vladlen Mykolaiovych Pakhomov, who was the first who used the transit calorimetry method to study the phase transformations of milk fat. 